\title{
Tracking $\mathrm{BO}_{6}$ Coupling in Perovskite Superlattices to Engineer Magnetic Interface Behavior
}

\author{
Qian $\mathrm{He}^{1}$, Saurabh Ghosh ${ }^{2}$, Eun Ju Moon ${ }^{3}$, Steven J. May ${ }^{3}$, Andrew R. Lupini, ${ }^{1}$ Sokrates T. Pantelides ${ }^{1,2}$,
} Albina Y. Borisevich ${ }^{1,4}$

1. Materials Science and Technology Division, Oak Ridge National Laboratory, Oak Ridge, TN, USA

2. Department of Physics and Astronomy, Vanderbilt University, Nashville, TN, United States

3. Department of Materials Science and Engineering, Drexel University, Philadelphia, PA, USA

4. The Center for Nanophase Materials Sciences, Oak Ridge National Laboratory, Oak Ridge, TN, USA

One of the fundamental topics in electron microscopy research is to understand heterointerfaces, where two different materials meet. Scanning transmission electron microscopes, with sub $\AA$ spatial resolution and pm level precision, are indispensable in these studies [1]. For instance, a measurement of atomic column positions and distances can identify the strain state of the thin film due to the epitaxy at the heterointerface. However, since the effective length scale for the epitaxial strain is either the pseudomorphic limit or film thickness, rarely less than tens of nm, such information can also be obtained by diffraction techniques (e.g. XRD). This is not the case for studying $\mathrm{BO}_{6}$ coupling behavior in perovskites due to symmetry mismatch at the heterointerfaces, due to a much smaller effective length scale of only a few unit cells [2].

In the past several years, control of $\mathrm{BO}_{6}$ coupling at perovskite interfaces has emerged as a new tool for engineering of interface properties [2] due to its strong coupling to polar and magnetic properties and even to oxygen conductivity [3]. High resolution data on tilt transitions at interfaces is instrumental for evaluating the validity of existing theoretical models and developing predictive theories. It is however a challenging experiment. Previous HRTEM [4], bright field (BF) [5] and annular bright field (ABF) [6] STEM work could only study one in-phase rotating axis at a time by locating oxygen columns in the images, therefore the full $3 \mathrm{D}$ rotation information was not available. 3D $\mathrm{BO}_{6}$ rotation information can be obtained from selected area electron diffraction (SAED) [7] but it suffers from dynamic scattering and limited resolution in thin film configuration; recently introduced position averaged converged beam electron diffraction (PACBED) [8] requires extensive simulations, which are especially prohibitive for materials with several competing phenomena, since each sample parameter such as thickness, polarization, strain, and tilt, adds an extra dimension to the simulation phase space.

Recently [9], we have developed a unique method to investigate $\mathrm{BO}_{6}$ rotation patterns in complex oxides $\mathrm{ABO}_{3}$ with unit cell resolution. Our method involves column shape analysis in ABF-STEM images of the $\mathrm{ABO}_{3}$ heterointerfaces taken in specific orientations. The rotating phase of $\mathrm{BO}_{6}$ octahedra can be identified for all three spatial dimensions without the need of case-by-case simulation. In several common rotation systems, quantitative measurements of all three rotation angles are now possible. For instance, the out-of-plane g rotation, whose axis is perpendicular to the beam direction, can be quantified using the major axis angle of the elliptical oxygen column image. (Figure 1a) Using this method, we examined interfaces between perovskites with distinct tilt systems as well as interfaces between tilted and un-tilted perovskites, identifying an unusual coupling behavior at the $\mathrm{CaTiO}_{3} / \mathrm{LSAT}$ interface. (Figure 1b-c)

This method will allow us to determine local symmetry between adjacent unit cells, revealing the $\mathrm{BO}_{6}$ coupling behavior at heterointerfaces in $3 \mathrm{D}$. The prospects of using this technique to characterize structure and predict properties will be demonstrated with a combined STEM and DFT study of magnetic superlattice of $\mathrm{La}(\mathrm{Ca}) \mathrm{MnO}_{3} / \mathrm{La}(\mathrm{Sr}) \mathrm{MnO}_{3}$ with different periodicities, which exhibit a range of electromagnetic coupling behaviors. We will also investigate the prospects for tilted structure determination using electron ptychography and evaluate its applicability on uniform and multi domain regions of the samples. The correlations among the $\mathrm{BO}_{6}$ rotation, domain size, superlattice periodicity and the electromagnetic coupling will be discussed in detail [10]. 


\section{References:}

[1] SJ Pennycook et al., MRS Bull. (2015).

[2] JM Rondinelli et al., MRS Bull. (2012)

[3] X Li et al., Chemistry of Materials (2015).

[4] AI Kirkland et al. Ultramicroscopy (2007).

[5] YM. Kim et al., Adv Mater (2013).

[6] E. Okunishi et. al., Micron (2012).

[7] DI Woodward et. al., Acta Crystallogr B (2005).

[8] J. Hwang et al., PRB (2013).

[9] Q. He et al., ACS Nano (2015)

[10] Research supported by the Materials Science and Engineering Division, U.S. Department of Energy (DOE), through a user project supported by ORNL's Center for Nanophase Materials Sciences, sponsored by the Scientific User Facilities Division, Office of Basic Energy Sciences, U.S. DOE.

(a)



(b) LSAT Сто

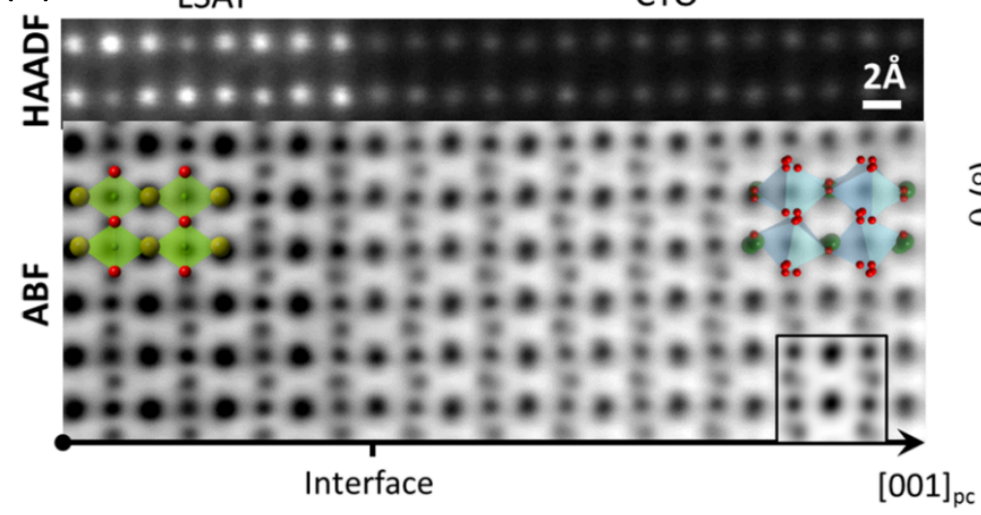

(c)

Figure 1. 3D information of the $\mathrm{BO}_{6}$ octahedral rotations in $\mathrm{ABO}_{3}$ perovskites from the shape analysis of oxygen columns. (a) Simulated ABF image chart of model perovskite structures with $\mathrm{BO}_{6}$ rotations of constant $\mathrm{a}$ and $\mathrm{b}$ but different $\mathrm{g}$. The overlapped polyhedral model highlights the position of oxygen columns (red). The major axis of the ellipse with respect to a reference (e.g. A-A or B-B) can be defined as q, which changes with the magnitude of the g rotation. (b) Experimental HAADF and ABF STEM images of a CaTiO3 (CTO) / LSAT interface, with overlapping polyhedral models and an inset of simulated image. (c) q profile measured from (b), showing a gradual change of the magnitude of the $g$ rotation in CTO, suggesting a presence of a new $\mathrm{BO}_{6}$ rotating pattern $\left(\mathrm{a}^{-}\right.$ $\mathrm{b}^{+} \mathrm{c}^{0}$ ) at the interface. (modified from ref 9 ). 